\title{
Les accords politiques du 18 octobre et 31 décembre 2016 en République Démocratique du Congo : entre sacrifice de la démocratie et gain de la paix ?
}

\author{
Phidias Ahadi Senge Milemba ${ }^{1}$
}

\begin{abstract}
This paper aimed to decipher the political agreements of October 18 and December 31, 2016 so as to finally screen their impact on democracy consolidation and on peace construction in Congo-Kinshasa. It demonstrates that those agreements have reversed the foundation of democracy and therefore consacrated its failure in favour of the satisfaction of the political actors and families' greed for power. Furthermore any attempt to construct peace through those political agreements has been illusive as long as citizens have been kept away from the political game where a new political regime devoid of strong popular support is setting in a tense political and social climate. Yet, we have to note from official speeches that we are living in a "democrativorous state".
\end{abstract}

Key words: Political agreement, democracy, egocentric power, dialogue, peace, sacrifice, "democrativorous state".

Résumé :- Ce papier avait pour toile de fond le décryptage des accords politiques du 18 octobre et 31 décembre 2016 pour, au final, passer au crible l'impact qu'ils arborent sur la consolidation de la démocratie et la construction de la paix. Il démontre que les désaccords entre la Majorité au pouvoir et l'Opposition sont source de tensions et d'instabilité de l'ordre juridique interne. En vue de leur règlement, les acteurs politiques ont, comme dans bien des pays en Afrique, recouru au dialogue avec, en filigrane, l'ambition d'accoucher d'un nouvel ordre juridique atypique et favorable à leur dessein. Ce droit né des ambitions politiques mesquines a, malencontreusement, renversé les fondements de la démocratie et rendu, contre toute attente, la paix illusoire. Pourtant, à en croire les discours officiels, nous vivons dans un « Etat démocrativore ».

Mots-clés : Accord politique, démocratie, dialogue, égocratie, "Etat démocrativore », paix, sacrifice.

\section{INTRODUCTION}

La démocratie est dans l'impasse en République Démocratique du Congo. Elle est aujourd'hui, plus que jamais, mise au premier rang des préoccupations de la conscience citoyenne, parce que, de l'avis de Francis Dupuis-Deri, a conservé la même définition pendant plus de deux mille ans, de la Grèce antique jusqu'au milieu du XIXe siècle, à savoir, un régime politique où le peuple se gouverne seul, sans autorité suprême qui puisse lui imposer sa volonté et le contraindre à l'obéissance ${ }^{2}$.

Elle est devenue un chantier toujours recommencé car, comme en 2003 avec l'entrée en fonction du gouvernement de transition issu des entendus de l'Accord du 17 décembre 2002, la population vient d'être de nouveau mise à l'écart des décisions politiques, à la suite des compromis de la Cité de l'Union Africaine et du Centre interdiocésain. Pourtant, elle venait de placer ses nouvelles attentes d'une gouvernance à la hauteur des défis de la société en l'organisation des élections prévues à la fin 2016. Le fait pour le pouvoir en exercice de n'avoir pas organisé les élections dans le délai constitutionnel, avait éveillé des suspicions populaires sur un probable glissement du régime en place bien au-delà de 2016 comme pour dire jeter du sang dans l'eau pour attirer le requin. On peut aussi rappeler la tentative d'adoption de la Loi électorale par l'Assemblée nationale, le samedi 17 janvier 2015, avec l'article 8 alinéa 3 très désapprouvé par une tranche populaire, lequel alinéas conditionnait la tenue des élections à l'organisation préalable du recensement général de la population.

Certains congolais, surtout ceux de l'Opposition, y ont descellé une manœuvre subtile visant à renvoyer l'élection présidentielle au-delà de 2016. Ces doutes ont vite cédé place aux ardeurs de rue qui, à leur tour, ont

${ }^{1}$ Professeur Associé, Faculté des Sciences Sociales, Administratives et Politiques, Département des Sciences Politiques et Administratives, Université de Goma, République Démocratique du Congo.

${ }^{2}$ F. Dupuis-Deri, Démocratie. Histoire politique d'un mot aux États-Unis et en France, Montréal, Lux éditeur, 2013, p.9. 
conduit aux manifestations violentes vécues du 19 au 21 janvier 2015 à Kinshasa et à l'intérieur du pays, attisées par les intermédiaires congolais intéressés soit par le devenir démocratique du pays soit par la soif du pouvoir.

Pour épargner le pays d'un lendemain chaotique et lui garantir la stabilité, fut signée l'ordonnance n¹5/084 du 28 novembre 2015 par le Président de la République, portant convocation du Dialogue Politique National Inclusif. Du coup, l'annonce de ce dialogue entraîna une ferveur réelle au sein de toute la classe politique et sociale congolaise. Cette ferveur traduit bien, pour un œil exercé, deux visions antithétiques. En premier lieu, un intérêt national rivé sur la recherche d'une sortie de crise apaisée, susceptible de sauvegarder des vies humaines et à éviter une destruction totale de ce que les congolais ont de commun, c'est-à-dire l'Etat. En second lieu, la résistance à la tenue dudit dialogue, car présumé une manœuvre subtile du régime en place de se pérenniser au pouvoir sans que l'organisation des élections dans le temps constitutionnel soit son cheval de batail.

Ces assises, ayant pris six mois pour démarrer, n'avaient pas, malheureusement, réuni tous les partis ténors de l'Opposition. D'où, la remise en cause de son caractère inclusif souhaité. Leurs conclusions ont été vite balayées par les puissants partis de l'Opposition réunis dans le Rassemblement des Forces Politiques et Sociales Acquis au Changement ayant boycotté ce fameux dialogue. Ainsi, seront-t-elles convoquées les deuxièmes assises sous l'œil-de-perdrix de l'Eglise catholique. Dans les premières assises comme dans les secondes, les peuples était mis hors-jeu politique. C'est le rapport des forces entre la Majorité et l'Opposition qui dictait la loi du système de gouvernance du pays.

Il n'est pas hors de propos de souligner ici, pour un esprit avisé, que toute tentative de la construction de la paix est illusoire tant que les citoyens resteraient gardés à l'écart du jeu politique au pays, où un nouveau régime politique est installé sans assise populaire puissante. Ainsi, le débat sur les accords politiques supposés gérer la période transitoire soulève deux questions de fond, auxquelles cette note propose des réponses. La première a trait au sacrifice de la démocratie pour le gain de la paix, tandis que la seconde s'intéresse aux bénéficiaires des compromis politiques de 2016.

Dans les lignes qui suivent, l'analyse sera centrée sur le décryptage des accords politiques en vue de déceler l'impact qu'ils arborent sur la consolidation de la démocratie et la paix au pays. Plus spécifiquement, il s'agira de comprendre dans quelle mesure les accords politiques signés sont porteurs d'espoir de la démocratie et de la paix.

\section{LES ACCORDS POLITIQUES CONTRE LA DEMOCRATIE}

La participation citoyenne à la vie politique est devenue, aujourd'hui, le baromètre de la santé démocratique d'un pays, parce que le citoyen en est la plaque tournante sans laquelle toute tentative de sa construction est utopique. A cet effet, il est mobilisé à chaque exercice démocratique et décide de divers niveaux de pouvoir dans la société. Le pouvoir part de lui et aboutit à lui, comme pour reprendre la formule de l'ancien chef d'Etat américain, Abraham Lincoln : «Le gouvernement du peuple, par le peuple et pour le peuple ${ }^{3} »$. La démocratie est, selon l'esprit de cette acception, « un pouvoir ensemble avec le peuple ${ }^{4}$ ».

En effet, prévues constitutionnellement à la fin 2016, les élections n'ont guère eu lieu dans cette temporalité, plutôt une machine politique, ici dénommée «Dialogue Politique National Inclusif », dont le principe de négociation est consubstantiel à l'activité humaine ${ }^{5}$, a été instituée pour décider, désormais, du devenir politique du pays. Ce détournement du principe démocratique, selon lequel la légitimité du pouvoir politique provient du souverain primaire, a d'évidents effets sur la gouvernance du pays ; parce que les assises politiques sont fonctions des calculs politiques coûts/avantages des acteurs en présence pour maximiser les positionnements ou repositionnements de leurs partis et regroupements politiques respectifs.

C'est à n'en point douter le marasme qui gangrène le pays, où la démocratie est écartée du système de gouvernance. On ne voit guère cette noble aspiration de la classe politique et sociale à ériger le pays en « Etat démocrativore ", c'est-à-dire élever le pays en ordre d'Etats caractérisés par un régime essentiellement démocratique et qui ne se nourrissent que de valeurs du même ordre, plutôt qu'une caricature d'Etat, dont la gestion est fondée sur le principe de consensualité entre signataires des accords politiques au mépris l'esprit de la Loi fondamentale, vitrine de la démocratie. D'où, l'exclusion du citoyen et strangulation de sa sensibilité active à la gestion des biens communs. Ce scénario de sortie de crise inspire le régime à pluralisme limité, mais non responsable à l'égard du peuple.

De plus, la démocratie fait aujourd'hui l'objet des désirs de plus en plus intransigeants de la part des citoyens. La demande pour une participation plus effective des populations aux affaires publiques a justifié les «consultations populaires » initiées par le président Mobutu en 1990 en vue de recueillir l'opinion que les

\footnotetext{
${ }^{3}$ J. Hallowel, Les fondements de la démocratie, Paris, Nouveaux Horizons, 1977, p.47.

${ }^{4} \mathrm{Ph}$. Ahadi Senge Milemba, «Les déterminants du vote au Congo-Kinshasa 2006-2011. Une contribution à la sociologie électorale », in African Journal of Democracy and Governance, Vol. 2, N² 2\&3, 2015, p.101.

${ }^{5}$ J.-L. Marret, La fabrication de la paix, nouveaux conflits, nouveaux acteurs, nouvelles méthodes, Paris, Ellipses, 2001, p.59. 
citoyens se faisaient sur le système de parti unique en place depuis 1965. Elle a également justifié et rendu possible la tenue entre 1991 et 1992 d'une «Conférence Nationale Souveraine », dont l'objectif était de faire le point sur l'état de la gouvernance dans le pays depuis son indépendance en 1960, et de jeter les bases d'un système nouveau de gouvernance débarrassé de la dictature du parti unique ${ }^{6}$.

Plus récemment encore, les manifestations populaires du 17 janvier 2015 dues à la tentative d'adoption de la Loi électorale par l'Assemblée nationale, dont l'article 8 alinéa 3 conditionnait la tenue des élections à l'organisation préalable du recensement général de la population ; les manifestations populaires vécues pendant trois jours au pays, soit du 19 au 21 janvier 2015, témoignent de l'aspiration intraitable des citoyens à participer plus effectivement aux affaires publiques, et selon Patrick Mignard, une crise de confiance des citoyens à l'égard des institutions de la République, et plus exactement à l'égard de la classe politique ${ }^{7}$.

On peut en douter, les accords politiques signés le 18 octobre et 31 décembre 2016 violent silencieusement la Constitution du 18 février 2006 sous un regard amorphe du peuple, enchainé derrière les idéologies des parties prenantes à ces pourparlers politiques. Parce qu'affirme Fodjo Kadjo Abo, on ne peut pas s'afficher comme un sauveur et se comporter en même temps comme un bourreau ${ }^{8}$. On relève ainsi, parmi les explications généralement fournies, que la naissance des compromis politiques, engendrés à la suite d'une crise ou d'une situation conflictuelle, advient surtout dans un contexte de contestation des institutions et des règles constitutionnelles ${ }^{9}$.

Le dialogue de la Cité de l'Union Africaine a eu lieu à Kinshasa sous la coupole d'un facilitateur désigné par l'Union Africaine, à savoir, Edem Kodjo. En effet, la tenue de ces assises, après une longue période d'attente et de suspens, est en soi un évènement : annoncées six mois au paravent, elles ont finalement démarré le $1^{\mathrm{er}}$ septembre 2016, alors que beaucoup de citoyens congolais ne croyaient plus à leur tenue et, de surcroit, n'y voyaient qu'un attrape pour calmer les ardeurs de rue face à la présomption d'un possible glissement du régime en place qui se dessinait peu à peu dans les nuages politiques congolais. La cérémonie de clôture avait eu lieu le 18 octobre 2015.

L'accord signé, a prévu l'élection présidentielle en avril 2018 et non à la fin 2016 comme constitutionnellement prévue. L'accord accorde également la primature à l'Opposition politique, contrairement à l'article 78 de la Constitution du 18 février 2006 qui dispose que : «Le Président de la République nomme le Premier ministre au sein de la majorité parlementaire après consultation de celle-ci. Il met fin à ses fonctions sur présentation par celui-ci de la démission du Gouvernement. Si une telle majorité n'existe pas, le Président de la République confie une mission d'information à une personnalité en vue d'identifier une coalition. La mission d'information est de trente jours renouvelable une seule fois. Le Président de la République nomme les autres membres du Gouvernement et met fin à leurs fonctions sur proposition du Premier ministre ${ }^{10}{ }$.

A ce titre, le Président en exercice est maintenu au pouvoir jusqu'à l'élection de son successeur. Cependant, l'accord ne précise pas qu'il ne peut se représenter ou soumettre la Constitution au referendum en vue de lever la limite du nombre de mandats. Tout de même, l'accord appelle au respect intégral des articles de la Loi des lois qui exclut ces deux options.

Pour les assises du Centre interdiocésain, celles-ci ont commencé le 8 décembre 2016 pour déboucher à la signature de l'accord le 31 décembre 2016. Des garanties telles que la non éligibilité du président en exercice aux élections à organiser à la fin 2017 ; l'exclusion d'une possible organisation du référendum constitutionnel ; la reconnaissance de la primature au Rassemblement des forces politiques et sociales acquis au changement...en ont résultées. Pour tout dire, l'article 78 de la Constitution de la République est de nouveau violé de plus belle par ce deuxième accord, et confirme l'idée de Pierre Calame, selon laquelle, nos sociétés semblent paralysées par l'acratie : cette maladie de la volonté qui fait que, sachant devoir changer, on ne trouve plus en soi-même la volonté de le faire ${ }^{11}$.

Tout compte fait, aux différentes phases de ces pourparlers, plusieurs acteurs, dont ceux de la Majorité, de l'Opposition, de la Société civile et de la Communauté international ont joué, chacun, un rôle distinct de

\footnotetext{
${ }^{6}$ Ngoma Binda, Otemikongo Mandefu et al., La RDC. Démocratie et participation à la vie politique : une évaluation des premiers pas dans la troisième République, Johannesburg, Afrimap et Open society initiative for Southern Africa, 2010, p.2.

${ }^{7} \mathrm{P}$. Mignard, L'illusion démocratique. Essai sur la politique à l'intention de celles et ceux qui doutent..., Toulouse, AAEL, 2003, p.10.

${ }^{8}$ Fodjo Kadjo Abo, La pratique de la terreur au nom de la démocratie, Paris, L’Harmattan, 2009, p.10.

${ }^{9}$ P. Mambo, « Les rapports entre la constitution et les accords politiques dans les États africains : Réflexion sur la légalité constitutionnelle en période de crise », in McGill Law Journal, Vol. 57, № 4, Juin 2012, p.926.

${ }^{10}$ Cabinet du Président de la République, «Constitution de la République Démocratique du Congo », in Journal Officiel, $47^{\text {̀̀me }}$ année, Numéro spécial, Kinshasa-18 février 2006.

${ }^{11} \mathrm{P}$. Calame, Sauvons la démocratie ! Lettre ouverte aux femmes et hommes politiques de notre temps, Paris, Editions Charles Léopold Mayer, 2012, p.7.
}

DOI: 10.9790/0837-2205066774 $\quad$ www.iosrjournals.org $\quad 69 \mid$ Page


l'autre, mais complémentaire dans la recherche d'une solution négociée à la crise électorale. Il y a eu, certes, regain de tension en des moments de débats rudes où des compromis étaient quasi-irréalisables de suite des attaques verbales, des préjugés sur l'autre ainsi que l'intransigeance des parties vis-à-vis de ce qu'elles ont des positions divergentes et infranchissables, ont rendu les pourparlers lents et longs. Ce qui a, maintes fois, provoqué des reports, des blocages et mêmes des échecs. Tout de même, elles ont fini par céder la place à des discussions qui ont abouti à la signature de l'accord du 18 octobre 2016 et plus tard, à celui du 31 décembre 2016. Les modalités d'application de ces accords n'ont guère trouvé, jusqu'alors, un terrain d'attente à cause de la fermeté des parties, de loin rivée sur l'intérêt en jeu : l'organisation des élections.

A tout bien comprendre, la signature des accords politiques marque la mise en ouvre d'un chantier politique de la réinvention d'une nouvelle gouvernance fondée sur la consensualité limitée, où les ambitions politiques ont écrasé le droit positif, un nouveau chantier sur lequel s'affrontent des intérêts mesquins au nom de la démocratie et la stabilité du pays, mais paradoxalement, dans un continuum d'évitement de la volonté du souverain primaire à participer à la gestion des affaires publiques. Ce constat amer est caractéristique de ce que Patrick Mignard appelle l' «illusion démocratique ${ }^{12}$ », un château ténébreux dans lequel toute la classe politique et sociale semble être prise à partie.

Pourtant, la nouvelle étincelle et le dynamisme revigoré, dont le peuple a pu dernièrement témoigner au monde le 17 janvier 2015, et deux jours plus tard encore, soit du 19 au 21 janvier 2015, donnent à croire qu'il jouera un rôle encore plus important à l'avènement d'un Etat démocrativore. Parce qu'aujourd'hui, en RD Congo, la culture politique du peuple a changé de manière significative. On ne doit rappeler à personne, les effets de demandes du peuple pour une participation plus effective aux affaires publiques depuis 1990. Le fait de contribuer à la construction d'une démocratie est positif pour tous, tant pour la Majorité au pouvoir que pour l'Opposition. Mener à bien cette lutte fera du peuple une ressource précieuse pour une fin démocratique attendue avec beaucoup d'impatience.

Tout de même, ces accords ont réussi à réunir au tour d'une même table les adversaires politiques et, par ricochet, atténuer les ferveurs de rue ainsi que celles des intermédiaires congolais, décidés à plonger le pays dans le chao si et seulement si le pouvoir en place s'en tenait à un autre mandat. La succession des compromis politiques en l'espace de trois mois a, en outre, permis de réorienter les désaccords et tensions au sein de la classe politique et sociale, en dépit des effets collatéraux que cela a pu entraîner dans l'ordonnancement juridique interne : le caractère anticonstitutionnel et extraconstitutionnel de certains articles des accords, au demeurant acceptés par les parties.

\section{LES ACCORDS POLITIQUES POUR UN GAIN ILLUSOIRE DE PAIX}

Naguère considérée comme un espace paisible, la RD Congo, un continent en miniature, est devenue depuis quelques décennies l'une des régions du globe à haut risque, une société à turbulence chronique forte, comme si les institutions politiques venaient de déclarer leur déchéance face à la montée incontrôlée de la criminalité odieuse. L'une des raisons de cette situation attendrissante est la perte de vitesse de la démocratie dans la gouvernance du pays, où ses chirurgiens l'ont maladroitement et bassement charcutée.

Le pays constitue, de ce fait, un mauvais exemple de la démocratie, au regard, dans bien des cas, des violations répétées de ses fondamentaux. Ce faible crédit de l'ordre juridique qui étale, en filigrane, la « démocratie émasculée ${ }^{13} \gg$, expliquerait la hantise, aujourd'hui chronique des Etats africains à s'en passer chaque fois que les intérêts privés divisent la classe politique. Ce système de gouvernance de nombre d'Etats en Afrique est bien moins « démocrativore » qu'elle ne laisse pas à la paix la chance de jaillir.

En effet, il est complexe que le renversement des fondements de la démocratie produise la paix ; que la signature des accords politiques, instituant les nouvelles institutions politiques incrustées de la crise de légitimité et du consensus, constitue « les rails qui mènent à la paix ${ }^{14}$ ». Certes, des mesures salutaires d'arrangement et de décrispation politiques contenues dans les annexes des accords sont un atout pour la stabilité, mais précaire parce que ne donnent pas de garanties suffisantes à la classe politique et sociale de fumer le calumet de la paix. Si mal gérées, ces mesures peuvent causer des retournements politiques difficiles à gérer surtout que le nouvel ordre juridique interne mis en place le 18 octobre comme celui du 31 décembre 2016 sont, dans le fond, en marge de l'esprit de la Constitution du 18 février 2006, et donc susceptibles de créer des zones d'instabilité dangereuses pour la santé politique du pays, dorénavant très fragile.

Ces accords politiques versus Constitution du 18 février 2006 constitueraient une réserve de tensions potentielles entre le gouvernement et le parlement. Une voie est donc ouverte à une possible activation d'une

\footnotetext{
${ }^{12}$ P. Mignard, Op. cit., p.1.

${ }^{13} \mathrm{~T}$. Holo, «Démocratie revitalisée ou démocratie émasculée ? Les constitutions du renouveau démocratique dans les États de l'espace francophone africain : régimes juridiques et systèmes politiques », in Revue béninoise des sciences juridiques et administratives, $\mathrm{N}^{\circ} 16,2006, \mathrm{p} .17$.

${ }^{14}$ Cette expression est empruntée de Fatou Diomé, Le Ventre de l'Atlantique, Paris, Anne Carrière, 2003 , p.294.

DOI: 10.9790/0837-2205066774 www.iosrjournals.org $\quad 70 \mid$ Page
}


crise de la démocratie parlementaire, car les accords proposent des dispositions qui affaiblissent l'action parlementaire sur le gouvernement. Par ce fait, le parlement se trouve estropié de ses prérogatives constitutionnelles sur l'exécutif. La situation de crise démocratique dans laquelle est mobilisé le principe de négociation, laisse, effet, augurer d'une réfutation du juridisme. L'appréhension du juridisme des accords politiques répond au besoin de stabilité que pourvoit le droit. Ce recours au droit laisse paraître la nécessité de normaliser une situation politique devenue catalyseur de l'instabilité des institutions politiques du pays. Il est donc nécessaire à plus d'un titre, de métrer consubstantiellement l'impact politique et juridique des accords au nouveau contexte politique, car le rapport entre la constitution et l'accord politique révèle une relation conflictuelle et sujet à débat inachevé ${ }^{15}$.

Le gouvernement étant l'émanation des accords politiques du 18 octobre et bientôt du 31 décembre 2016, un ordre politique créé à la circonstance pour gérer la conjoncture politique et juridique, l'article 78 de la Constitution congolaise sort l'une des premières victimes à être sacrifiée sur l'autel des négociations politiques. Par ce fait, les compromis politiques du 18 octobre et 31 décembre 2016 hypothèquent la Constitution du 18 février 2006 et illico presto se présentent comme supérieurs à celle-ci par leurs dispositions novatrices qui lui sont contraires.

A tout prendre, les rails qui mènent à la paix sont en piteux état ${ }^{16}$, et ne garantissent pas une paix stable. Parce que tout apprentissage de construction de la paix est chimérique tant que le peuple resterait à ce point mis à l'écart du cercle des décisions politiques. C'est pourquoi, affirme l'ancien Secrétaire général des Nations Unies, Boutros Boutros-Ghali, la démocratie contribue à préserver la paix et la sécurité, la justice sociale et les droits de l'homme et à promouvoir le développement économique et social ${ }^{17}$. L'expérience a également montré que, tout comme le prix de l'exclusion n'est autre que la violence, bien souvent l'inclusion de tous dans la vie politique est un gage de stabilité ${ }^{18}$.

Irrécusablement, la démocratie est prise au piège des accords politiques qui risquent d'impacter fatalement sur le fonctionnement des institutions politiques de la transition ; car elle est le seul cadre permettant de gérer sur le long terme des intérêts [...] concurrents tout en réduisant au minimum le risque d'un conflit interne violent. De plus, en assumant une plus forte participation populaire, la démocratie permet de définir les objectifs de développement tenant compte des grandes aspirations et priorités de la société ${ }^{19}$. La nouvelle fenêtre d'ouverture à exploiter, aujourd'hui, réside dans la voie négociée, dont les accords politiques paraissent une clé possible du dénouement de la crise électorale congolaise si et seulement si ne s'érige pas en tortionnaire de l'esprit et la lettre de la Constitution du 18 février 2006.

Cependant, ces négociations politiques, au lieu de porter sur le diagnostic réel de la crise qu'est l'organisation des élections, ont plutôt placé dans leur angle de tir la partie de l'iceberg qui émerge de l'eau, l'invisible étant abordé superficiellement. Ainsi, une question mérite bien d'être posée. Est-il possible qu'un malade dont la température est supérieure à $38{ }^{\circ} \mathrm{C}$ guérisse avec un traitement à base de l'eau froide, sans au préalable poser un diagnostic des causes de cette hyperthermie ? En pareil cas, où les pronostics priment sur les diagnostics cliniques, les complications sont susceptibles de remonter à la surface pour finalement ralentir ou tout simplement bloquer le rétablissement du patient.

En effet, cette base tronquée des négociations des accords constitue un potentiel de sa remise en cause par l'une ou l'autre partie, car affirme Innocent Ehueni Manzan, la mauvaise écriture qui caractérise des dispositions de certains accords politiques n'est pas de nature à assurer une application efficace et efficiente en ce qu'elle donne lieu à diverses interprétations confuses voire contradictoires de la part des signataires, chacun l'interprétant en sa faveur au détriment de l'autre ${ }^{20}$.

On le voit, tous ces accords signés en vue de fumer le calumet de la paix ne sauront empêcher la réapparition des germes d'une possible activation de la crise par une difficile cohabitation de l'exécutif avec le législatif d'une part, et le conformisme attendu des institutions de transition à la Constitution du 18 février 2006 et, selon le cas, aux accords du 18 octobre et 31 décembre 2016 d'autre part, deux ordres juridiques internes antithétiques et potentiels sources d'un conflit juridique. Parce qu'affirme Paterne Mambo, la relation entre la

\footnotetext{
${ }^{15}$ Pour approfondissement, lire P. Mambo, Ibid. ; J.-L. Atangana Amougou, « Les accords de paix dans l'ordre juridique interne en Afrique », in Droit prospectif : Revue de la recherche juridique, Vol. 33, $\mathrm{N}^{\circ} 123,2008$, pp.1723-1746.

${ }^{16}$ A. Nzadi-a-Nzadi, «Les rails qui mènent à la paix durable en Afrique», in Congo-Afrique, $\mathrm{N}^{\circ} 510$, décembre 2016, p.917.

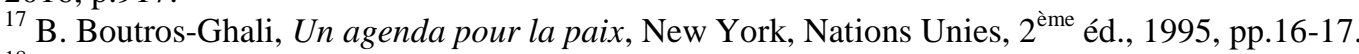

${ }^{18}$ Rapport du Secrétaire général, document ONU 58/212, 4 aout 2003, paragraphe 2. Cité par G.S. Goodwi-Gill, Elections libres et régulières, Nouvelle édition augmentée, Genève, Union interparlementaire, 2006, p.18.

${ }^{19}$ B. Boutros-Ghali, Op. cit., pp.120-128.

${ }^{20}$ I. Ehueni Manzan, Les accords politiques dans la résolution des conflits armés internes en Afrique, Thèse de doctorat en Droit, Paris, Université de La Rochelle/Université de Cocody-Abidjan, 2011, p.618.

DOI: 10.9790/0837-2205066774 www.iosrjournals.org $\quad 71 \mid$ Page
}


constitution et les arrangements politiques est souvent caractérisée par des rapports conflictuels et contradictoires. Cette conflictualité conduit au renversement de la pyramide des normes, et favorise en outre la déstabilisation de l'ordre constitutionnel. On constate ainsi une déconstruction de la logique du positivisme classique $^{21}$.

De plus, il est difficile voire impossible de constater une bonne négociation dès lors que les participants demeurent ancrés dans une psychologie de rejet de l'autre et dans un état d'esprit de bourreau et/ou de victime tendant à justifier ses propres agissements en condamnant ceux de la partie ennemie ${ }^{22}$. Du coup, la mobilisation d'un discours d'insatisfaction dans le partage des responsabilités gouvernementales, le mode de désignation du chef du gouvernement, du président du Conseil National du Suivi de l'Accord accompagné des actes de méfiance entre compétiteurs politiques de tendances politiques opposées, feront que l'instabilité s'en sorte grandie.

\section{LE PEUPLE : BENEFICIAIRE DE FAÇADE DES ACCORDS POLITIQUES}

L'expression «accord politique » est riche de synonyme dans une variabilité de contexte : voie de sortie de crise, compromis politiques, accord de paix, arrangements politiques particuliers, conventions politiques, etc. L'étude ne prétend nullement investir le débat querellé entre spécialistes sur les nuances synonymiques de la notion. Néanmoins, elle retient l'expression « accord politique » à partir du moment où la réalité exprimée par les autres expressions sus évoquées est, à quelque différence près, la même dans ce papier.

De ce fait, réfléchissant à l'importance que la Majorité au pouvoir et l'Opposition en RD Congo en particulier et en Afrique en général, ont toujours attachée aux manœuvres de sortie d'une situation de conjoncture politique basse, l'accord politique est devenu un enjeu pompeux pour éviter une fin tragique à un régime politique, truffé de secousses sociopolitiques chroniques. Ainsi, l'accord politique porterait le menu de la réunion des volontés, la conformité des sentiments des individus (acteurs politiques, acteurs sociaux) et/ou des groupes (Majorité, opposition, société civile) aux fins de créer l'effet de droit voulu dans la mutation de l'ordre juridique interne existant.

A tout prendre, les accords politiques sont fonctions des calculs politiciens, proportionnellement au poids politique des acteurs et/ou groupes en présence en vue de la maximisation des (ré) positionnements politiques des uns et des autres. Ils font prévaloir la justesse et la noblesse de leur vue sans, généralement, respecter l'ordre juridique interne existant. Cependant, cette institutionnalisation, si elle ne respecte pas le jeu démocratique voulu par le peuple, reste, malencontreusement, un moyen sournois d'instauration d'un système de gouvernance châtrée de la démocratie. Du coup, ces accords auraient à consacrer le sinistre de la démocratie, car privant le peuple de son désir intransigeant de vivre l'expérience de l'alternance démocratique protégée par la constitution.

A vrai dire, les délégués aux dialogues du 18 octobre comme ceux du 31 décembre 2016 ne s'y trouvaient pas avec un agenda de relève du défi démocratique au nom de l'intérêt supérieur de la nation. Chaque délégué jouait à la défensive des intérêts de son regroupement politique, reléguant ainsi au second plan l'intérêt supérieur de la nation. Aussi, se sont-ils empressés à se partager le gâteau gouvernemental sans se soucier de l'alternance démocratique, pourtant un des aspects fondamentaux de l'épine dorsale de la crise politique congolaise. Cette situation illustre clairement que la question de l'intérêt de la nation n'est pas à l'avant plan des discussions politiques entreprises depuis le $1^{\mathrm{er}}$ septembre 2016, plutôt, constitue le plus grand commun diviseur parmi les opérateurs politiques à la Cité de l'Union Africaine et au Centre interdiocésain.

C'est pourquoi, affirme Kamana Tshibengabo, la RD Congo est, certes, un « grand pays », mais bien malade de ses fils et filles. La cause principale de cette situation catastrophique présente, une cause aussi lointaine qu'actuelle, se trouve dans le déficit du patriotisme [...]. A voir nos comportements de tous les jours, nous devons avouer que nous n'avons jamais donné suffisamment d'affection à notre pays pour lui permettre de se hisser dans le concert des nations et d'y maintenir vigoureusement sa place. « Mon pays avant tout », semble n'avoir jamais été un slogan préféré des Congolais ${ }^{23}$.

Comme on le voit, à ces négociations dures, le peuple est le plus grand perdant des accords politiques, parce que mis à l'écart des décisions sur l'avenir du pays, et par-dessus tout pris en otage par l' «égocratie ${ }^{24}$ » camouflée de ceux qui revêtissent la politique congolaise de la peau de chagrin. Alors que le peuple venait de placer toute sa confiance en eux pour apporter la lumière au système de gouvernance du pays, de mener des

${ }^{21}$ P. Mambo, Op. cit., pp. 927-928.

${ }^{22}$ Idem, p.132.

${ }^{23}$ Kamana Tshibengabo, République Démocratique du Congo : la défense nationale à l'impératif. Patriotisme et Souveraineté, Coll. «Points de vie », Paris, L'Harmattan, 2004, p.1.

${ }^{24}$ Concept signifiant, pour certains, la personnalisation du pouvoir parmi les représentants du peuple, ce papier l'entend plutôt comme un pouvoir dont le peuple n'est pas l'émanation par un des modes civilisés d'acquisition du pouvoir. Lire pour approfondissement A. Martin, Egocratie et démocratie. La nécessité de nouvelles technologies politiques, Limoges, Fypéditions, 2010.

\begin{tabular}{lll}
\hline DOI: $10.9790 / 0837-2205066774$ & www.iosrjournals.org & $72 \mid$ Page
\end{tabular}


négociations éclairées et de peser de tout leur poids pour faire entendre la raison, les acteurs politiques se sont plutôt éperdument intéressés à savoir qui de la Majorité au pouvoir ou de l'Opposition prendra les manettes de commande du gouvernement de transition? Quelle taille aura-t-il en vue d'assouvir leur soif du pouvoir? Qui présidera le Conseil National du Suivi de l'Accord ? De combien de membres la plénière du Conseil National du Suivi de l'Accord sera-t-elle composée ? Comment seront repartis les portefeuilles de l'Etat?

Par ce fait, l'accord a produit plus de rivalités que de compromis entre parties contestateurs. A tout bien comprendre par analogie, dans notre société, ce qui permet à l'homme de connaître, de juger et d'agir conformément à des principes (la raison) semble avoir cédé place à l'aliénation : cette maladie psychique qui rend l'individu comme étranger à lui-même et à la société fait que, sachant que fumer est préjudiciable à la santé, on ne trouve plus en soi-même la volonté d'arrêter.

L'objectif intrinsèque poursuivi par les acteurs serait l'accumulation des ressources financières en particulier, en cette période vouée à la crise de la démocratie parlementaire, où le législatif n'aura pas une tâche facile à contrôler l'exécutif. Ces ressources permettraient le financement de leurs campagnes électorales aux élections prévues à la fin 2017, dont la tenue est désormais à placer dans le registre de l'incertitude, au regard de la coupable lenteur des parties à tirer unanimement les rideaux des arrangements particuliers à l'accord.

On peut s'en apercevoir, la RD Congo peine jusqu'aujourd'hui à trouver des acteurs politiques patriotes et démocrates qui permettraient la transcendance des intérêts mesquins, des intérêts partisans au profit de l'intérêt supérieur de la nation et de la démocratie. Le détournement de la lutte politique patriotique et démocratique au profit de la politique d'abreuvoir présente une menace à la démocratie et à la paix dans le macrocosme politique congolais. Tout laisse croire que, la RD Congo a ardemment besoin des patriotes véritables, non pas de «ventriotes » ventripotents à la bonne mine une fois dans un épisode politique de mangeoire ; besoin des leaders éclairés, actifs et animés avant tout par la volonté irréprochable de servir la démocratie et transformer notre société, et non de martyrs pour le besoin de l'histoire.

\section{CONCLUSION}

La République Démocratique du Congo est devenue un pays démocratiquement sinistré. La signature des accords politiques y est devenue, dans bien des cas, un moyen atypique d'éjection du citoyen du cercle de décision politique. Ce renversement des fondements de la démocratie à des fins politiques égocratiques est on ne peut plus déplorable dans le chantier démocratique congolais. Il consacre, à cet effet, la noyade de la démocratie dans les eaux mortifères au profit de la satisfaction des intérêts politiques privés, dans un continuum de mutation de l'ordre juridique interne, taillée sur mesure des ambitions politiques des acteurs.

Avec le naufrage de l'ordre constitutionnel établi depuis le 18 février 2006 au profit d'un autre, fruit de la volonté des politiques à la Cité de l'Union Africaine et au Centre interdiocésain, la météorologie politique semble indiquer que l'application effective de l'accord du 31 décembre avance déjà vers un état de profonde incertitude. Ce à quoi on peut déjà s'attendre dans l'horizon politique congolais est que les effets positifs de l'application de l'accord seront inférieurs aux coûts du détournement des fondements de la démocratie.

Il est donc urgent et pressant que le système de gouvernance de la RD Congo devienne «démocrativore » afin de susciter et nourrir son peuple d'un espoir pour un lendemain de paix et de développement. Parce qu'il est illusoire d'espérer vivre la paix dans un contexte très peu voué aux valeurs intrinsèques de la démocratie, et où la relation entre la loi-mère et les conventions politiques est d'une nature atypique $^{25}$ au regard de bien des dispositions légales délibérément réfutées au profit des arrangements politiques cautionnés par les parties.

Ce raisonnement permet donc de porter un nouveau regard sur l'intérêt supérieur de la nation qui, en démocratie, doit l'emporter sur les critères individualistes des gestionnaires des institutions, des partis ou regroupements politiques, avec comme conséquence, affirme Clayton Thomas, des décisions plus efficaces, un public satisfait et solidaire et surtout une démocratie renforcée ${ }^{26}$. S'il arrive que l'accord du 31 décembre 2016 n'est guère effectivement mis en œuvre par les parties qui l'ont signé, ce n'est pas tant la mauvaise foi des parties, mais parce que l'accord en soi n'est pas assorti de règles obligatoires et juridiquement sanctionnées visà-vis des parties récalcitrantes. Cette situation est, dans bien des cas, le catalyseur de la perte de considération des dispositions des accords politiques et consacre, par ce fait, son affaiblissement continu.

Par ailleurs, le dialogue reste un ingrédient de taille pour prévenir et/ou traiter le déséquilibre et tensions dans la gouvernance du pays. Cependant, il ne doit pas être source d'une situation atypique pour sacrifier la démocratie ainsi que la paix. Il ne doit non plus se substituer à l'ordre juridique établi avec la participation des citoyens éclairés ou le modifier en sa défaveur. S'il importe de dialoguer, et à terme signer un

\footnotetext{
${ }^{25}$ Lire pour approfondissement P. Mambo, Op. cit., pp. 921-952.

${ }^{26}$ J. Clayton Thomas, Action publique et participation des citoyens. Pour une gestion démocratique revitalisée, Paris, Nouveaux Horizons, 1995, p.2.

DOI: 10.9790/0837-2205066774 www.iosrjournals.org $\quad 73 \mid$ Page
}


accord politique, celui-ci doit se conformer à l'esprit et à la lettre de la constitution pour que la démocratie et la paix s'en sortent garanties.

\section{BIBLIOGRAPHIQUES}

[1] Ahadi Senge Milemba Ph., «Les déterminants du vote au Congo-Kinshasa 2006-2011. Une contribution à la sociologie électorale », in African Journal of Democracy and Governance, Vol. 2, $\mathrm{N}^{\circ} 2 \& 3,2015$, pp.97-111.

[2] Atangana Amougou J.-L., « Les accords de paix dans l'ordre juridique interne en Afrique », in Droit prospectif : Revue de la recherche juridique, Vol. 33, N¹23, 2008, pp.1723-1746.

[3] Boutros-Ghali B., Un agenda pour la paix, New York, Nations Unies, 2 ème éd., 1995.

[4] Cabinet du Président de la République, «Constitution de la République Démocratique du Congo », in Journal Officiel, $47^{\text {ème }}$ année, Numéro spécial, Kinshasa - 18 février 2006.

[5] Calame P., Sauvons la démocratie! Lettre ouverte aux femmes et hommes politiques de notre temps, Paris, Editions Charles Léopold Mayer, 2012.

[6] Clayton Thomas J., Action publique et participation des citoyens. Pour une gestion démocratique revitalisée, Paris, Nouveaux Horizons, 1995.

[7] Dupuis-Déri, F., Démocratie. Histoire politique d'un mot aux États-Unis et en France, Montréal, Lux Éditeur, 2013.

[8] Ehueni Manzan I., Les accords politiques dans la résolution des conflits armés internes en Afrique, Thèse de doctorat en Droit, Paris, Université de La Rochelle/Université de Cocody-Abidjan, 2011.

[9] Fatou Diomé, Le Ventre de l'Atlantique, Paris, Anne Carrière, 2003.

[10] Fodjo Kadjo Abo, La pratique de la terreur au nom de la démocratie, Paris, L'Harmattan, 2009.

[11] Goodwi-Gill G.S., Elections libres et régulières, Nouvelle édition augmentée, Genève, Union interparlementaire, 2006.

[12] Hallowel J., Les fondements de la démocratie, Paris, Nouveaux Horizons, 1977.

[13] Holo T., « Démocratie revitalisée ou démocratie émasculée ? Les constitutions du renouveau démocratique dans les États de l'espace francophone africain : régimes juridiques et systèmes politiques », in Revue béninoise des sciences juridiques et administratives, $\mathrm{N}^{\circ} 16,2006, \mathrm{pp} .17-41$.

[14] Kamana Tshibengabo, République Démocratique du Congo: la défense nationale à l'impératif. Patriotisme et Souveraineté, Coll. « Points de vue », Paris, L’Harmattan, 2004.

[15] Mambo P., «Les rapports entre la constitution et les accords politiques dans les États africains : Réflexion sur la légalité constitutionnelle en période de crise », in McGill Law Journal, Volume 57, $\mathrm{N}^{\circ}$ 4, Juin 2012, pp. 921-952.

[16] Marret J.-L., La fabrication de la paix, nouveaux conflits, nouveaux acteurs, nouvelles méthodes, Paris, Ellipses, 2001.

[17] Martin A., Egocratie et démocratie. La nécessité de nouvelles technologies politiques, Limoges, Fypéditions, 2010.

[18] Mignard P., L'illusion démocratique. Essai sur la politique à l'intention de celles et ceux qui doutent..., Toulouse, AAEL, 2003.

[19] Ngoma Binda, Otemikongo Mandefu et al., La RDC. Démocratie et participation à la vie politique : une évaluation des premiers pas dans la troisième République, Johannesburg, Afrimap et Open society initiative for Southern Africa, 2010.

[20] Nzadi-a-Nzadi A., «Les rails qui mènent à la paix durable en Afrique», in Congo-Afrique, N510, décembre 2016, pp.917-918. 